\title{
Mac Lane, Bourbaki, and Adjoints: A Heteromorphic Retrospective
}

\author{
David Ellerman \\ Philosophy Department, University of California at Riverside
}

\begin{abstract}
Saunders Mac Lane famously remarked that "Bourbaki just missed" formulating adjoints in a 1948 appendix (written no doubt by Pierre Samuel) to an early draft of Algebre-which then had to wait until Daniel Kan's 1958 paper on adjoint functors. But Mac Lane was using the orthodox treatment of adjoints that only contemplates the object-to-object morphisms within a category, i.e., homomorphisms. When Samuel's treatment is reconsidered in view of the treatment of adjoints using heteromorphisms or hets (object-to-object morphisms between objects in different categories), then he, in effect, isolated the concept of a left representation solving a universal mapping problem. When dualized to obtain the concept of a right representation, the two halves only need to be united to obtain an adjunction. Thus Samuel was only a now-simple dualization away for formulating adjoints in 1948. Apparently, Bodo Pareigis' 1970 text was the first and perhaps only text to give the heterodox "new characterization" (i.e., heteromorphic treatment) of adjoints. Orthodox category theory uses various relatively artificial devices to avoid formally recognizing hets-even though hets are routinely used by the working mathematician. Finally we consider a "philosophical" question as to whether the most important concept in category theory is the notion of an adjunction or the notion of a representation giving a universal mapping property (where adjunctions arise as the special case of a bi-representation of dual universal mapping problems).
\end{abstract}

\section{Contents}

1 Introduction $\quad 2$

2 A primer on homs and hets $\quad 2$

3 Mac Lane on Bourbaki and adjunctions 2

4 Left and right representations of Het-bifunctors $\quad 4$

5 Pareigis' "new characterization" of an adjunction 5

$\begin{array}{llr}6 & \text { Samuel-with dualization } & 7\end{array}$

$\begin{array}{llr}7 & \text { Chimeras in the wilds of mathematical practice } & 8\end{array}$

8 Heterophobic devices: Mac Lane's universal arrows $\quad 9$

9 The tensor product as an important example $r 9$

10 Cones and cocones as hets 11

11 Adjoints vs. UMPs: What's basic? 13

12 Conclusions $\quad 15$ 


\section{Introduction}

Saunders Mac Lane famously said the "Bourbaki just missed" [20, Notes at end of Adjoints chapter] defining adjunctions in a 1948 Appendix III "On Universal Mappings" to an early version of Algebre Chapter III on multilinear algebras [3]. The Appendix was no doubt written by Pierre Samuel who published a very similar paper on under his own name the same year [26] and was even referenced in the Appendix so we will "pierce the veil" of Bourbakian anonymity and henceforth refer to Samuel as the author.

A heterodox treatment of adjunctions (Pareigis [25]; rediscovered and developed in Ellerman [9]) using heteromorphisms shows that Samuel may have been even closer than Mac Lane surmised. The heteromorphic treatment splits an adjunction (as "molecule") into two "atoms" which are solutions to dual universal mapping problems. Mac Lane shows how to formulate Samuel's universal mapping property (UMP) in categorical terms, and it is precisely a solution to one of the universal problems in the heteromorphic treatment. Thus it only needs to be dualized (turning the mappings around) to arrive at the notion of a solution to the dual universal problem. Putting the two UMP solutions together yields the notion of an adjunction a decade before Kan's definition [13] (which does not use heteromorphisms). ${ }^{1}$

\section{A primer on homs and hets}

In the conventional approach to category theory, the only object-to-object maps are between objects in the same category and thus they are properly called homomorphisms or homs for short. The intuitive idea of a heteromorphism (het for short) is an object-to-object morphism between the objects of different categories like the injection-of-generators morphism $x \rightarrow F(x)$ from a set $x$ to the free group $F(x)$ generated by $x$. Since hets have their tail in one category and head in another, they may also be colorfully called chimera morphisms. Hets should not be confused with functors which map one whole category to another. For instance, the free group functor $F: S e t \rightarrow G r p$ from the category of sets $S e t$ to the category of groups Grp has the action on Set to take a set $x$ to a group $F(x)$, often symbolized $x \longmapsto F(x)$, which is quite distinct from the set-to-group injection-of-generators heteromorphism $x \rightarrow F(x)$.

Heteromorphisms between two given categories can be treated using a bifunctor analogous to the way homomorphisms are treated within a category. When one is "given" a category $X$, then it is assumed that includes the composition of homs within the category and that is specified rigorously by a Hombifunctor Hom : $X^{o p} \times X \rightarrow$ Set. Given an $X$-hom $x^{\prime} \stackrel{f}{\Longrightarrow} x$ in $\operatorname{Hom}\left(x^{\prime}, x\right)$, then its composition with another $X$-hom $x^{\prime \prime} \stackrel{f^{\prime}}{\Longrightarrow} x^{\prime}$ is given by the induced map $\operatorname{Hom}\left(f^{\prime}, x\right): \operatorname{Hom}\left(x^{\prime}, x\right) \rightarrow \operatorname{Hom}\left(x^{\prime \prime}, x\right)$ where $\operatorname{Hom}\left(f^{\prime}, x\right)(f)=x^{\prime \prime} \stackrel{f^{\prime}}{\Longrightarrow} x^{\prime} \stackrel{f}{\Longrightarrow} x$.

Just as one assumes the Hom-bifunctor for the morphisms of a category when "given" that category, so when one uses heteromorphisms as part of the category-theoretic data, then when "given" two categories $X$ and $A$, then one would assume given not only two Hom-bifunctors $\operatorname{Hom}_{X}: X^{o p} \times X \rightarrow$ Set and $\operatorname{Hom}_{A}$ : $A^{o p} \times A \rightarrow$ Set, but also the two Het-bifunctors Het $\operatorname{H}_{X A}: X^{o p} \times A \rightarrow$ Set and $\operatorname{Het}_{A \rightarrow X}: A^{o p} \times X \rightarrow$ Set.

The analogous way in which Het-bifunctors describe the composition of hets and homs is explained in the next section in the context of Mac Lane's comments about Bourbaki.

\section{Mac Lane on Bourbaki and adjunctions}

Consider Mac Lane's historical comments on Bourbaki and adjoints.

One may also speculate as to why the discovery of adjoint functors was so delayed. ... Bourbaki just missed ([1948], Appendix III). His definition of universal construction was clumsy, because

\footnotetext{
${ }^{1}$ The long and somewhat contentious-or at least storied-relationship between Mac Lane and Bourbaki is considered at length in Corry [6] and the references contained therein. For more about Mac Lane, see McLarty [24]. For the orthodox homs-only treatment of adjoints, see in addition to Mac Lane [20], McLarty [23], Awodey [1], or Marquis [22].
} 
it avoided categorical language, but it amounted to studying a bifunctor $W: X^{o p} \times A \longrightarrow$ Set and asking for a universal element of $W(x,-)$ for each $x$. This amounts to asking for objects $F x \in A$ and a natural isomorphism $W(x, a) \cong A(F x, a)$; it includes the problem of finding a left adjoint $F$ to a functor $G: A \longrightarrow X$, with $W(x, a)=\operatorname{hom}_{X}(x, G a)$... Bourbaki's construction problem emphasized representable functors, and asked "Find $F x$ so that $W(x, a) \cong A(F x, a)$ ". This formulation lacks the symmetry of the adjunction problem, "Find $F x$ so that $X(x, G a) \cong$ $A(F x, a)^{\prime \prime}$ - and so missed a basic discovery; this discovery was left to a younger man, perhaps one less beholden to tradition or to fashion. [20, p. 108]

Mac Lane reviews the book containing that Appendix III in 1949 [19], but then only mentions without further comment the appendix on the "universal mapping" problem. That Appendix III was dropped in the final edition of Algebre [5], although some of the content appears (first published in 1957) in the section on Universal Mappings in Bourbaki's Elements of Sets [4].

Mac Lane noted in Categories for the Working Mathematician (first published in 1971) that Bourbaki's (i.e., Samuel's) statement of the universal mapping problem "amounted to" using a bifunctor

$$
W: X^{o p} \times A \rightarrow \text { Set }
$$

and then for each $x$, asking for a representation in $\mathbf{A}$ of the functor $W\left(x,{ }_{-}\right)$. Bodo Pareigis calls such a bifunctor a connection [25, p. 58] from category $X$ to category $A$; today such a bifunctor is also called a Set-valued profunctor [14], distributor [2], or correspondence [17, p. 96] depending on the context. The elements of $W(x, a)$ can be interpreted as heteromorphisms or hets from a object $x$ in $X$ to an object $a$ in $A .^{2}$

The Het-bifunctor gives the rigorous way to handle the composition of a het $\varphi: x \rightarrow a$ in $W(x, a)$ [thin arrows $\rightarrow$ and Greek letters for hets] with a homomorphism or hom $g: x^{\prime} \Longrightarrow x$ in $X$ [thick Arrows $\Longrightarrow$ and Roman letters for homs] and a hom $h: a \Longrightarrow a^{\prime}$ in $A$. For instance, the composition $x^{\prime} \stackrel{g}{\longrightarrow} x \stackrel{\varphi}{\rightarrow} a$ is the het that is the image of $\varphi$ under the map: $W(g, a): W(x, a) \rightarrow W\left(x^{\prime}, a\right)$. Similarly, the composition $x \stackrel{\varphi}{\rightarrow} a \stackrel{h}{\Longrightarrow} a^{\prime}$ is the het that is the image of $\varphi$ under the map: $W(x, h): W(x, a) \rightarrow W\left(x, a^{\prime}\right) .^{3}$

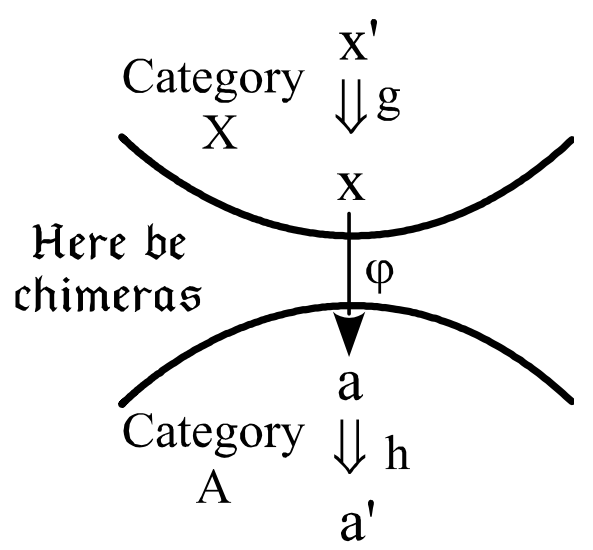

Composition of a het with a hom on either end

\footnotetext{
${ }^{2}$ The concrete or intuitive interpretation as heteromorphisms of the elements in the values of a "naked" bifunctor Het : $X^{o p} \times A \rightarrow$ Set is not available for the bifunctors defined from given functors going one way or another between the categories such as: $\operatorname{Hom}_{X}(F(x), a)$ or $\operatorname{Hom}_{A}(x, G(a))$ (including comma categories, e.g., Mac Lane [20, p. 45]) since those are clearly hom-sets within a category. However, such bifunctors with hom-set values appear when there is a left or right representation of a naked bifunctor Het : $X^{o p} \times A \rightarrow$ Set.

${ }^{3}$ The definition of a bifunctor also insures the associativity of composition so that schematically: hom $\circ($ het $\circ$ hom $)=$ (hom $\circ$ het) $\circ$ hom.
} 
This is all perfectly analogous to the use of Hom-functors to define the composition of homs, and thus the bifunctor with the het interpretation of its elements is best termed a Het-bifunctor Het : $X^{o p} \times A \rightarrow$ Set (normal arrows $\rightarrow$ for all functors).

\section{Left and right representations of Het-bifunctors}

Given the het bifunctor Mac Lane referred to as $W(x, a)$, the universal mapping problem for each $x \in X$ is: does there exist an object $F(x) \in A$ and a canonical het $\eta_{X}: x \rightarrow F(x)$ such that given any het $\varphi: x \rightarrow a$ (to any $a \in A$ ), there exists a unique hom $\underline{f}: F(x) \Longrightarrow a$ such that the following diagram commutes?

$$
\begin{array}{ccc}
x & & \varphi \\
\eta_{x} \downarrow & \searrow & \\
F(x) & \stackrel{\searrow}{\exists ! \underline{f}} & a
\end{array}
$$

Universal mapping problem

If there is a solution for each $x \in X$, that is equivalent to the Het-bifunctor $W(x, a)$ being represented on the left by a functor $F: X \rightarrow A$, which means for each object $x \in X$, there is an isomorphism natural in $a$ :

$$
\begin{gathered}
\operatorname{Hom}_{\mathbf{A}}(F(x), a) \cong W(x, a) \\
\text { Left representation. }
\end{gathered}
$$

That is said to be a left representation of the Het-bifunctor $W(x, a)$ or left representation for short ("universal solution of the universal problem" in Pareigis [25, p. 58]). A left representation is a "universal mapping situation" that gives the functor solution of the het-version of a UMP and often gives half of an adjoint situation (e.g., "half-adjunction" [9]) in which case the left-representing functor $F$ is the left adjoint.

Instead of taking $W(x, a)$ as being independently defined as a Het-functor, Mac Lane notes that this would define a left adjoint to a given functor $G: A \rightarrow X$ if we defined $W(x, a):=\operatorname{Hom}_{\mathbf{X}}(x, G(a))$. Having thus introduced the asymmetry by defining $W(x, a)$ in this manner already assuming $G$, Mac Lane goes on to say that Bourbaki missed the symmetry of the adjunction equivalence, where $F(x)$ gives a left representation of the bifunctor $\operatorname{Hom}_{\mathbf{X}}(x, G(a))$. But given the ("naked") Het-functor $W(x, a)$ (i.e., without any $G$ ), the obvious symmetric thing to do is to dually define a functor $G: A \rightarrow X$ from the representation of $W(\ldots, a)$ on the right so for each $a \in A$, there is an isomorphism natural in $x$ :

$$
\begin{gathered}
W(x, a) \cong \operatorname{Hom}_{\mathbf{X}}(x, G(a)) \\
\text { Right representation }
\end{gathered}
$$

which might be called a right representation of the Het-bifunctor $W(x, a)$ or right representation for short.

The dual co-universal mapping problem for each $a \in A$ is: does there exist an object $G(a) \in X$ and a canonical het $\varepsilon_{a}: G(a) \rightarrow a$ such that for any het $\varphi: x \rightarrow a$ (from any $x \in X$ ), there exists a unique hom $\bar{f}: x \Longrightarrow G(a)$ such that the following diagram commutes?

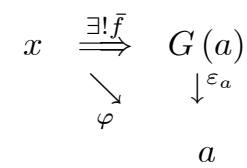

Co-universal mapping problem

A solution for every $a \in A$ defines right representing functor $G: A \rightarrow X$ that gives the right representation.

If the Het-bifunctor is represented symmetrically on both the left and right, then we have the natural isomorphisms of an adjunction equivalence: 


\section{$\operatorname{Hom}_{\mathbf{A}}(F(x), a) \cong W(x, a) \cong \operatorname{Hom}_{\mathbf{X}}(x, G(a))$}

Heteromorphic treatment of an adjunction

where $F(x)$ and $G(a)$ are respectively the left and right adjoints. The canonical het unit $\eta_{x}: x \rightarrow F(x)$ is obtained by taking $a=F(x)$ on the left and then taking the image of $1_{F(x)}$ in $W(x, F(x))$ under the isomorphism. The canonical het counit $\varepsilon_{a}: G(a) \rightarrow a$ is obtained by taking $x=G(a)$ on the right and then taking the image of $1_{G(a)}$ in $W(G(a), a)$ under the isomorphism. Then the adjunction is represented very simply by the adjunctive square diagram.

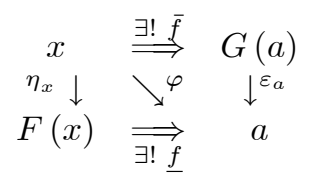

Adjunctive square diagram

Given any het $\varphi: x \rightarrow a$, the left isomorphism gives the unique hom $f: F(x) \Rightarrow a$ so the lower triangle commutes and the right isomorphism gives the unique hom $\bar{f}: x \Rightarrow G(\bar{a})$ so the upper triangle commutes. ${ }^{4}$

All this is perfectly symmetrical and it considerably simplifies the convoluted "over-and-back diagrams" for the conventional homs-only treatment of adjoints where given a hom $f: x \Rightarrow G(a)$ in one category, there exists a unique hom $f: F(x) \Rightarrow a$ in the other category so that the image hom $G(f): G(F(x)) \Rightarrow G(a)$ back in the original category make the following diagram commute. ${ }^{5}$

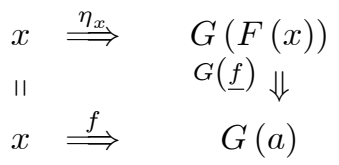

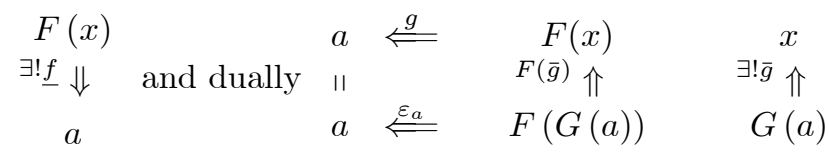

Homs-only diagrams for an adjunction

Contrary to Mac Lane's remarks, the het treatment is equally or more symmetric as the conventional homs-only treatment and even simpler. Mac Lane in effect gives Samuel credit for isolating the left representation that solves a universal mapping problem. But the turn-the-arrows-around category-theoretic duality was not clearly understood in 1948 so Samuel did not arrive at the symmetric dual formulation and solution to the co-universal mapping problem, namely a right representation. ${ }^{6}$ Thus we might say that Samuel was only a dualization away from defining adjoints in 1948 since adjoints arise as the left and right representing functors for the hets $W(x, a)$ going from a category $X$ to a category $A$.

\section{Pareigis' "new characterization" of an adjunction}

Bodo Pareigis's 1970 text, Categories and Functors [25] (translation of the 1969 German edition), is to our knowledge the first text and, to this day, the only category theory text that in effect uses a Het-bifunctor (called a "connection") to give the heteromorphic "new characterization for pairs of adjoint functors" [25, p. 60]. Pareigis does not call the elements of $\operatorname{Het}_{X \rightarrow A}(x, a)$ [or in his notation: $\operatorname{Mor}_{\mathcal{V}}(A, B)[25$, p. 58] where $A \in \mathcal{C}$ and $B \in \mathcal{D}$ ] heteromorphisms. Indeed, he quickly defines a new category $\mathcal{V}(\mathcal{C}, \mathcal{D})$ whose objects are the disjoint union of the objects of the categories $\mathcal{C}$ and $\mathcal{D}$, and whose morphisms are of three kinds, the homs

\footnotetext{
${ }^{4}$ There is an interesting side issue; do adjoints $F: X \rightleftarrows A: G$ have a "directionality" between the categories $X$ and $A$ ? That is far from clear in the usual treatment since the functors go both ways and all the object-to-object morphisms are homs within the two categories. But in the adjunctive square diagram, all the hets go in the same direction from $X$ to $A$.

${ }^{5}$ We have used the standard Greek letters $\eta_{x}: x \Rightarrow G(F(x))$ and $\varepsilon_{a}: F(G(a)) \Rightarrow a$ for the usual unit and counit even though they are homs.

${ }^{6}$ It is something of a mystery why Mac Lane in 1971 [20, Notes at end of Adjoints chaper] did not consider the dual right representation of Samuel's Het-bifunctor $W(x, a)$ instead of starting with a functor $G: A \rightarrow X$ and the resulting bifunctor $\operatorname{Hom}_{X}(x, G(a))$ and then pointing out that the left representation would yield an adjunction: $\operatorname{Hom}_{A}(F(x), a) \cong$ $\operatorname{Hom}_{X}(x, G(a))$.
} 
of $\mathcal{C}$, the hets from $\mathcal{C}$ to $\mathcal{D}$, and the homs of $\mathcal{D}$-all of which are seen as "homs" in $\mathcal{V}(\mathcal{C}, \mathcal{D})$. Pareigis calls it a directly connected category $\mathcal{V}(\mathcal{C}, \mathcal{D})$ with the connection $\operatorname{Mor}_{\mathcal{V}}(A, B)$. Given a bifunctor $H: \mathcal{C}^{o p} \times \mathcal{D} \rightarrow S e t$, this category today might be called the cograph [27] of the bifunctor and denoted $\mathcal{C} \star^{H} \mathcal{D}\left[17\right.$, p. 96]. ${ }^{7}$

From the first formalizations of a "universal mapping problem" in 1948 (Samuel [26]; Bourbaki [3]), the hets $W(x, a)=\operatorname{Het}_{X \rightarrow A}(x, a)$ (for a given $x \in X$ ) have been an intrinsic part of the data, since the "solution" $F(x)$ to the universal problem is a (left) representation of those hets by homs $\operatorname{Hom}_{A}(F(x), a)$ within the codomain category $A[25$, Lemma 2, p. 58].

Basic Idea: Universal mapping situations are about universally representing the hets from one category to another within one of the categories ("half" an adjunction) or within both categories (adjunction).

For the original universal mapping problem, a solution for each $x \in X$ yields a functor $F: X \rightarrow A$ giving the natural isomorphism:

$$
\operatorname{Hom}_{X}(F(x), a) \cong \operatorname{Het}(x, a)
$$

or in Pareigis' notation:

$$
\operatorname{Mor}_{\mathcal{D}}(\mathcal{F}(A), B) \cong \operatorname{Mor}_{\mathcal{V}}(A, B) \text {. }
$$

The dual co-universal mapping problem (which Samuel did not formulate) starts with the same het bifunctor data $W(x, a)=\operatorname{Het}_{X \rightarrow A}(x, a)$ and asks, for a given $a \in A$, for a (right) representation $G(a)$ of those hets by homs $\operatorname{Hom}_{X}(x, G(a))$ within the domain category $X$.

In Pareigis' (perhaps unnecessarily complicated) presentation of the dual case, he starts with a "connection" going the other way, i.e., a bifunctor $\mathcal{D}^{o p} \times \mathcal{C} \rightarrow \operatorname{Set}$, so that $\mathcal{V}(\mathcal{D}, \mathcal{C})$ is the corresponding directly connected category. He also denotes this category as $\mathcal{V}^{\prime}(\mathcal{C}, \mathcal{D})$ and calls it an inversely connected category [25, p. 60 where "inversely" is mis-spelled as "universely."]. The co-universal problem is, for a given $A \in \mathcal{C}$ :

Is there an object $U(A) \in \mathcal{D}$ and a morphism $\rho_{A}: U(A) \rightarrow A$ such that for each morphism $g: B \rightarrow A$ for all $B \in \mathcal{D}$ there is exactly one morphism $g^{*}: B \rightarrow U(A)$ making the diagram

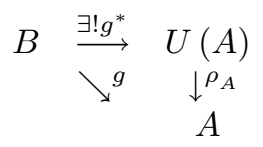

commutative? [25, p. 60]

A solution for every $A \in C$ would again define a representing functor $U(-)$.

Then Pareigis arrives at his "new characterization" of an adjunction by starting with the connection $\operatorname{Mor}_{\mathcal{V}}(A, B)$ so that $\mathcal{V}(\mathcal{C}, \mathcal{D})$ is directly connected and $\mathcal{V}^{\prime}(\mathcal{D}, \mathcal{C})$ [note the reversal in the arguments from the previous $\left.\mathcal{V}^{\prime}(\mathcal{C}, \mathcal{D})\right]$ is inversely connected. Then both the universal problems have solutions for all $A \in \mathcal{C}$ and $B \in \mathcal{D}$ if and only if we have a pair of adjoint functors $\mathcal{F}: \mathcal{C} \rightarrow \mathcal{D}$ and $\mathcal{G}: \mathcal{D} \rightarrow \mathcal{C}$ with the natural isomorphisms between the morphism sets [25, Theorem 2, pp. 60-1.]:

$$
\operatorname{Mor}_{\mathcal{D}}(\mathcal{F}(A), B) \cong \operatorname{Mor}_{\mathcal{V}}(A, B) \cong \operatorname{Mor}_{\mathcal{C}}(A, \mathcal{G}(B))
$$

Adjunction equivalence with the het middle term.

Pareigis did not splice the two triangular diagrams for the universal and co-universal problems together at their common hypotenuse, but doing so would yield the commutative diagram (with our notation for the canonical hets):

\footnotetext{
${ }^{7}$ If $\{0 \leq 1\}$ is the ordered set considered as a category with $0 \rightarrow 1$, then Jacob Lurie points out that these categories are in oneto-one correspondence with the functors $\chi: M \rightarrow\{0 \leq 1\}$ for any category $M\left[17\right.$, p. 97]. Given a bifunctor $H: \mathcal{C}^{o p} \times \mathcal{D} \rightarrow S e t$, there is the obvious functor $\chi: \mathcal{C} \star^{H} \mathcal{D} \rightarrow\{0 \leq 1\}$, and given such a functor $\chi: M \rightarrow\{0 \leq 1\}$, take $\mathcal{C}=\chi^{-1}(0), \mathcal{D}=\chi^{-1}(1)$, and for $A \in \mathcal{C}$ and $B \in \mathcal{D}$, the Het-bifunctor is given by $\operatorname{Het}_{\mathcal{C} \rightarrow \mathcal{D}}(A, B)=\operatorname{Hom}_{M}(A, B)$.
} 


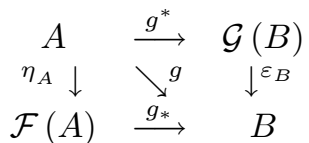

Adjunctive square diagram.

Pareigis' text shows that the heteromorphic treatment of adjunctions dates from at least the late 1960's, but it has not "caught on" and is, to our knowledge, not used in any of the more recent texts. Hence it remains an eccentric or heterodox treatment that is not part of the "official" orthodoxy. In spite of the informal use of hets in mathematical practice, various devices are used in the orthodox formal treatment of universal problems and adjoints so that hets are not explicitly recognized. Contrary to that later orthodoxy, Samuel formally recognized hets in his 1948 statement of the universal problem, but he missed getting Pareigis' heteromorphic characterization of adjoints a decade before Kan gave the orthodox treatment since Samuel did not develop the dual co-universal problem.

\section{Samuel-with dualization}

Pierre Samuel was explicit about the treatment of homs and hets and denoted them respectively with Roman and Greek letters (and we have here followed, for the most part, that Samuel notational convention). He worked with structured sets such as $S$-sets and $T$-sets so the homs would be the $S$-mappings or $T$-mappings. He called the hets from an $S$-set to a $T$-set "( $S$-T)-mappings" [26, p. 592] and he denoted them with Greek letters to recognize their difference from homs. Samuel also noted that the composition of a $(S-T)$-mapping with a $T$-mapping would be a $(S-T)$-mapping, i.e., hets composed with homs are hets. Samuel ([3, Appendix III] and [26]) used a standard scheme for a solution to a universal problem where given an $S$-set $E$, there is a "canonically associated" (old language for a functor) $T$-set $F_{0}$ and a canonical $\left(S\right.$ - $T$ )-mapping $\varphi_{0}: E \rightarrow F_{0}$ such that for any other $\left(S\right.$-T)-mapping $\varphi: E \rightarrow F$, there is a uniquely determined $T$-mapping $f: F_{0} \Rightarrow F$ such that the following diagram commutes.

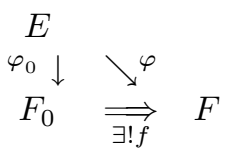

Samuel's universal mapping problem and solution.

Samuel did not see that this scheme could be dualized as a solution to a co-universal problem as follows. Given a $T$-set $F$, there is a canonically associated $S$-set $E^{0}$ and a canonical $\left(S\right.$ - $T$ )-mapping $\varphi^{0}: E^{0} \rightarrow F$ such that for any $\left(S\right.$-T)-mapping $\varphi: E \rightarrow F$, there is a uniquely determined $S$-mapping $g: E \Longrightarrow E^{0}$ such that the following diagram commutes.

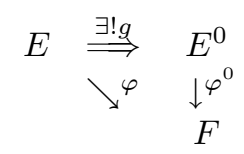

Dual of Samuel's scheme.

Splicing the two triangular diagrams together at the common diagonal het $\varphi$ gives Samuel's "almost" definition of an adjunction.

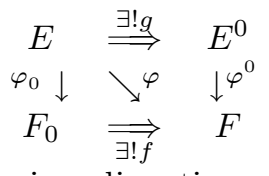

Samuel's near-miss adjunctive square diagram. 


\section{Chimeras in the wilds of mathematical practice}

The homs-only orientation may go back to the original conception of category theory "as a continuation of the Klein Erlanger Programm, in the sense that a geometrical space with its group of transformations is generalized to a category with its algebra of mappings." [7, p. 237] While chimeras do not appear in the orthodox "ontological zoo" of category theory, they abound in the wilds of mathematical practice. In spite of the reference to "Working Mathematician" in the title of Mac Lane's text [20], one might seriously doubt that any working mathematician would give, say, the universal mapping property of free groups using the "device" of the underlying set functor $U$ instead of the traditional description given in the left representation diagram (which does not even mention $U$ ) as can be seen in most any non-category-theoretic text that treats free groups. For instance, consider the following description in Nathan Jacobson's text [12, p. 69].

To summarize: given the set $X=\left\{x_{1}, \ldots, x_{r}\right\}$ we have obtained a map $x_{i} \rightarrow \bar{x}_{i}$ of $X$ into a group $F G^{(r)}$ such that if $G$ is any group and $x_{i} \rightarrow a_{i}, 1 \leq i \leq r$ is any map of $X$ into $G$ then we have a unique homomorphism of $F G^{(r)}$ into $G$, making the following diagram commutative:

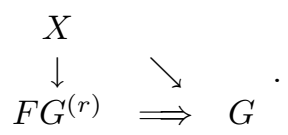

In Jacobson's diagram, only the $F G^{(r)} \Longrightarrow G$ morphism is a group homomorphism; the vertical and diagonal maps are sets-to-groups hets so it is the diagram for a left representation. ${ }^{8}$

Indeed, it might be interesting to see how Mac Lane handled the hets naturally involved in a universal mapping property before the development of the homs-only devices used later. To characterize the product within a category $C$, a het $c \rightarrow\langle a, b\rangle$ from an object $c$ in the category $C$ to an object $\langle a, b\rangle$ in the category $C \times C$ is a pair of $C$-morphisms $f: c \Rightarrow a$ and $g: c \Rightarrow b$ which Mac Lane called a "system" of maps [18, p. $264]$ and was later called a "cone" (a common type of het or chimera morphism). ${ }^{9}$ Fixing $\langle a, b\rangle$, the product $a \times b$ is given by a right representation. That is, there is a universal het $a \times b \rightarrow\langle a, b\rangle$ consisting of the cone-het of $C$-homs $p_{a}: a \times b \Rightarrow a$ and $p_{b}: a \times b \Rightarrow b$ (the projections) from $a \times b$ to $\langle a, b\rangle$ such that for any cone-het $f: c \Rightarrow a$ and $g: c \Rightarrow b$ from an object $c$ in $C$ to the object $\langle a, b\rangle$ in the different category $C \times C$, there is a unique $C$-hom $c \rightarrow a \times b$ such that the $C$-hom followed by the universal het equals the given het. Thus the $C$-hom $c \Rightarrow a \times b$ is the unique factor map that "transfers" (in this case, "reflects") the property of "being a cone $(f, g): c \rightarrow\langle a, b\rangle$ " from the universal such cone-het (the projections) to the given such cone $f: c \Rightarrow a$ and $g: c \Rightarrow b$ (Ellerman [8]). That is the het statement of the universal mapping property for the product. A het-free description of the product would be as a right adjoint to a diagonal functor $\Delta: C \rightarrow C \times C$ using the homs-only treatment of adjoints (see the later section on cones and cocones as hets for the general treatment) .

Diagonal functors and forgetful functors are two "devices" used to give an adjoint characterization of various significant functors without using hets, when there is a simpler characterization available using hets. For instance, Mac Lane [20, p. 87] gives a long list of forgetful functors whose left adjoints are significant mathematical constructions. Using the heteromorphic treatment, those significant left adjoints can all be obtained as the left representing functors without mentioning the device of the forgetful functor-as is typical in mathematical practice. Similarly Mac Lane [20, pp. 87-8] gives a long list of significant functors that are the left or right adjoints to diagonal functors, but again in the heteromorphic treatment, the significant functors can be characterized as the left or right representing functors without using the device of a diagonal functor. When not giving "official" statements, category theorists, as working mathematicians, routinely use hets (e.g., cones and cocones) informally, and in some cases, have to use hets to state the universal mapping characterization of a functor (e.g., the case of tensor products considered below).

\footnotetext{
${ }^{8}$ We modified Jacobson's diagram according to our het-hom convention for the arrows. Other examples from Mac LaneBirkhoff's text [21] are given later.

${ }^{9}$ Thus the values of Het $(c,\langle a, b\rangle)$ can be identified with the Cartesian product $\operatorname{Hom}_{C}(c, a) \times \operatorname{Hom}_{C}(c, b)$.
} 


\section{Heterophobic devices: Mac Lane's universal arrows}

One het-avoidance device is just to avoid the middle term in adjunction equivalence:

$$
\operatorname{Hom}_{A}(F(x), a) \cong \operatorname{Het}(x, a) \cong \operatorname{Hom}_{X}(x, G(a))
$$

and to use the homs-only over-and-back adjunction diagrams. While Samuel, Bourbaki, Pareigis, and working mathematicians routinely use hets in stating universal mapping properties, Mac Lane went to some lengths to give a het-free definition of a universal arrow from an object to a functor [20, p. 55].

Given the forgetful functor $U: \mathbf{C a t} \longrightarrow \mathbf{G r p h}$ and a graph $G$, we have constructed (§ II.7) the free category $C$ on $G$ and the morphism $P: G \longrightarrow U C$ of graphs which embeds $G$ in $C$, and we have shown that this arrow $P$ is "universal" from $G$ to $U$. A similar universality property holds for the morphisms embedding generators into free algebraic systems of other types, such as groups or rings. Here is the general concept.

Definition. If $S: D \longrightarrow C$ is a functor and $c$ an object of $C$, a universal arrow from $c$ to $S$ is a pair $(r, u)$ consisting of an object $r$ of $D$ and an arrow $u: c \longrightarrow S r$ of $C$, such that to every pair $(d, f)$ with $d$ an object of $D$ and $f: c \longrightarrow S d$ an arrow of $C$, there is a unique arrow $f^{\prime}: r \longrightarrow d$ of $D$ with $S f^{\prime} \circ u=f$. In other words, every arrow $f$ to $S$ factors uniquely through the universal arrow $u$, as in the commutative diagram

$\begin{array}{cccc}c & \stackrel{u}{\longrightarrow} & S r & r \\ \| & & \downarrow S f^{\prime} & \downarrow^{f^{\prime}} \\ c & \stackrel{f}{\longrightarrow} & S d & d .\end{array}$

For an example, take $c=x$ as the set of generators of the free group $r=F(x)$ and $S=U$ as the underlying set functor with $d$ as any other group $G$. Then the universal arrow is $x \Longrightarrow U(F(x))$ so for any $f: x \Rightarrow U(G), \exists ! \underline{f}: F(x) \Rightarrow G$ such that:

$\begin{array}{lccr}x & \stackrel{\eta_{x}}{\Rightarrow} & U(F(x)) & F(x) \\ \| & & U(\underline{f}) \Downarrow & \exists ! \underline{f} \Downarrow \\ x & \stackrel{f}{\Rightarrow} & U(G) & G\end{array}$

Homs-only over-and-back diagram for free-group/underlying-set adjunction.

Thus the free group universal mapping property is stated as a universal arrow from the set of generators to the underlying set functor $U$ from Grp to Set.

The free group functor $F(x)$ can be more naturally characterized as the left representing functor for the bifunctor Het : Set ${ }^{o p} \times G r p \rightarrow$ Set where the elements in Het $(x, G)$ are all the set-to-group functions $\varphi: x \rightarrow G$. The corresponding diagram for the heteromorphic treatment is that the free group functor is a left representation diagram for the hets $(\rightarrow)$ from a fixed set $x$ to any group $G$.

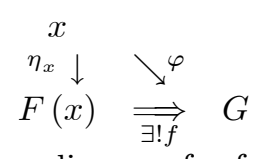

Left representation diagram for free-group functor.

\section{The tensor product as an important example}

In Mac Lane's remarks about Bourbaki-Samuel, he notes the special case of the tensor product functor. 
[Samuel's universal problem] also includes the problem of finding a tensor product for two modules $A$ and $B$, with $W(<A, B>, C)$ taken to be the set of bilinear functions $A \times B \rightarrow C$. Moreover, the tensor product $A \otimes B$ is not in this way an example of a left adjoint (though it is an example of our universal arrows).[20, p. 108]

The universal mapping property of the tensor product is of interest since it is a case where the heteromorphic treatment is forced since, as Mac Lane notes, the tensor product functor $\otimes:\langle A, B\rangle \longmapsto A \otimes B$ is not a left adjoint ${ }^{10}$ and thus the usual device of using the other adjoint (e.g., a forgetful functor) to avoid hets is not available.

For $A, B, C$ modules (over some commutative ring $R$ ), one category is the product category $\operatorname{Mod}_{R} \times$ $\operatorname{Mod}_{R}$ where the objects are ordered pairs $\langle A, B\rangle$ of $R$-modules and the other category is just the category $\operatorname{Mod}_{R}$ of $R$-modules. The values of the Het-bifunctor Het $(\langle A, B\rangle, C)$ are the bilinear functions $A \times B \rightarrow C$. Then the tensor product functor $\otimes: \operatorname{Mod}_{R} \times \operatorname{Mod}_{R} \rightarrow \operatorname{Mod}_{R}$ given by $\langle A, B\rangle \longmapsto A \otimes B$ gives a left representation:

$$
\operatorname{Hom}_{M o d}(A \otimes B, C) \cong \operatorname{Het}(\langle A, B\rangle, C)
$$

that characterizes the tensor product. The canonical het $\eta_{\langle A, B\rangle}: A \times B \rightarrow A \otimes B$ is the image under the isomorphism of the identity hom $1_{A \otimes B}$ obtained by taking $C=A \otimes B$, so we have:

$$
\begin{array}{ccc}
\langle A, B\rangle & & \\
\eta_{\langle A, B\rangle} \downarrow & \searrow^{\varphi} & \\
A \otimes B & \underset{\exists ! \underline{f}}{\Longrightarrow} & C
\end{array}
$$

Left representation diagram to characterize tensor products

where the single arrows are the bilinear hets and the thick Arrow is a module homomorphism within the category $\operatorname{Mod}_{R}$.

Since the tensor product functor is not a left adjoint, Mac Lane defines the notion of a universal element to give a seemingly het-free treatment.

The idea of universality is sometimes expressed in terms of "universal elements". If $D$ is a category and $H: D \longrightarrow$ Set a functor, a universal element of the functor $H$ is a pair $\langle r, e\rangle$ consisting of an object $r \in D$ and an element $e \in H r$ such that for every pair $\langle d, x\rangle$ with $x \in H d$ there is a unique arrow $f: r \longrightarrow d$ of $D$ with $(H f) e=x$. [20, p. 57]

As Mac Lane noted [20, p. 58], this can be diagrammed as a universal arrow (from $\{*\}$ to $H$ ) using "the" one-element set $\{*\}$ and a set function $\ulcorner x\urcorner:\{*\} \Rightarrow H(d)$ to pick out an element $x \in H(d)$, and which involves only homs in $D$ or Set.

$$
\begin{array}{cccr}
\{*\} & \stackrel{\ulcorner e\urcorner}{\Longrightarrow} & H(r) & r \\
\| & & H(f) \Downarrow & \exists ! f \\
\{*\} & \stackrel{\ulcorner x\urcorner}{\Longrightarrow} & H(d) & d
\end{array}
$$

Then Mac Lane notes that the bilinear universal mapping property for the tensor product can be shoe-horned into this homs-only notion of a universal element.

\footnotetext{
${ }^{10}$ The "nearest" adjunction is the linear version of the Currying adjunction obtained by fixing $B$ :
}

$$
\operatorname{Hom}_{M o d}(A \otimes B, C) \cong \operatorname{Hom}_{M o d}\left(A, \operatorname{Hom}_{M o d}(B, C)\right) .
$$


Tensor products provide another example of universal elements. Given two vector spaces $V$ and $V^{\prime}$ over the field $K$, the function $H$ which assigns to each vector space $W$ the set $H W=$ $\operatorname{Bilin}\left(V, V^{\prime} ; W\right)$ of all bi-linear functions $V \times V^{\prime} \longrightarrow W$ is the object function of a functor $H: \operatorname{Vect}_{K} \longrightarrow$ Set, and the usual construction of the tensor product provides both a vector space $V \otimes V^{\prime}$ and a bilinear function $\otimes: V \times V^{\prime} \longrightarrow V \otimes V^{\prime}$, usually written $\left\langle v, v^{\prime}\right\rangle \longmapsto v \otimes v^{\prime}$, so that the pair $\left\langle V \otimes V^{\prime}, \otimes\right\rangle$ is a universal element for the functor $H=\operatorname{Bilin}\left(V, V^{\prime} ;-\right)$. This applies equally well when the field $K$ is replaced by a commutative ring (and vector spaces by $K$-modules). [20, p. 58]

In this example (using $R$-modules instead of vector spaces), the Het-functor is: Bilin : $\left(\operatorname{Mod}_{R} \times \operatorname{Mod}_{R}\right)^{o p} \times$ $\operatorname{Mod}_{R} \rightarrow$ Set so fixing $\langle A, B\rangle, H(C)=\operatorname{Het}(\langle A, B\rangle, C)$ and then the above diagram for the universal element is:

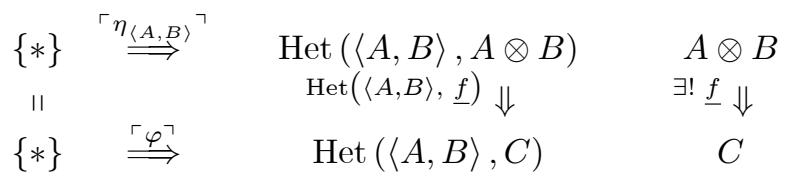

which is another convoluted (over-and-back) and seemingly het-free restatement of the simple left representation diagram for the tensor product given above. In the diagram, Mac Lane avoids explicit reference to the hets by using the Het-bifunctor itself $\operatorname{Het}(\langle A, B\rangle, C)$ explicitly to handle the het-hom composition: $A \times B \stackrel{\eta_{\langle A, B\rangle}}{\longrightarrow} A \otimes B \stackrel{f}{\longrightarrow} C=A \times B \stackrel{\varphi}{\longrightarrow} C$ as $\operatorname{Het}(\langle A, B\rangle, \underline{f})\left(\eta_{\langle A, B\rangle}\right)=\varphi \in \operatorname{Het}(\langle A, B\rangle, C)$, where $H(C)=\operatorname{Het}(\langle A, B\rangle, C)$ is just the Het-bifunctor holding $\langle A, B\rangle$ fixed. Thus the rather jerryrigged notion of a universal element uses the Het-bifunctor $H(C)=\operatorname{Bilin}(\langle A, B\rangle, C)$ and the set-homs $\{*\} \stackrel{\left\ulcorner\eta_{\langle A, B\rangle}\right\urcorner}{\Longrightarrow} \operatorname{Het}(\langle A, B\rangle, A \otimes B)$ and $\{*\} \stackrel{\ulcorner\varphi\urcorner}{\Longrightarrow} \operatorname{Het}(\langle A, B\rangle, C)$ to avoid mentioning the images of $*$ which are the hets $\left\ulcorner\eta_{\langle A, B\rangle}\right\urcorner(*)=\eta_{\langle A, B\rangle}$ and $\ulcorner\varphi\urcorner(*)=\varphi$.

In Mac Lane and Birkhoff's Algebra textbook [21], they explicitly use hets starting with the special case of a $K$-module $A$ (for a commutative ring $K$ ) and then stating the universal mapping property of the tensor product $A \otimes K \cong A$ using the left representation diagram [21, p. 318]-like any other working mathematicians. They do not use the homs-only universal element diagram; instead they compose (without comment) the universal bilinear het $h_{0}$ with a linear transformation hom $t$ between $K$-modules: $A \times K \stackrel{h_{0}}{\longrightarrow} A \stackrel{t}{\Longrightarrow} C$ which is equal to the given bilinear het $A \times K \stackrel{h}{\rightarrow} C$.

In other words, the arbitrary bilinear function $h$ is expressed as a composite $h=t \circ h_{0}$ with the fixed bilinear function $h_{0}$, as in the commutative diagram

$$
\begin{array}{cc}
A \times K \stackrel{h_{0}}{\underset{h}{h}} & A \\
& \Downarrow^{t} \\
& C
\end{array}
$$

(where we have used our notation for the hom $\Downarrow^{t}$ in the diagram).

\section{Cones and cocones as hets}

One type of het that routinely occurs, without formal recognition as a het, is a cone or cocone. Consider the device of the diagonal functor $\Delta: C \rightarrow C^{J}$ where $J$ is a "diagram" functor category and $C$ has limits to give the usual adjunction of the limit functor as the right adjoint to the diagonal functor:

$$
\operatorname{Hom}_{C^{J}}(\Delta c, d) \cong \operatorname{Hom}_{C}(c, \lim d) .
$$


The "cones" are hets going from an object $c \in C$ to a functor $d \in C^{J}$; formally a cone $\varphi: c \rightarrow d$ is a set $\left\{f_{j}\right\}_{j \in J}=\{c \Rightarrow d(j)\}_{j \in J}$ of $C$-homs indexed by the objects $j \in J$ such that for each $J$-hom $h: j \Rightarrow j^{\prime}$,

$$
c \Longrightarrow d(j) \stackrel{d(h)}{\Longrightarrow} d\left(j^{\prime}\right)=c \Longrightarrow d\left(j^{\prime}\right) .
$$

The Het-bifunctor Het : $C^{o p} \times C^{J} \rightarrow$ Set takes $\langle c, d\rangle$ to the set of cones from $c$ to $d$. The composition for $C$-homs $f: c^{\prime} \rightarrow c$ is obvious so consider a $C^{J}$-hom $\eta: d \Longrightarrow d^{\prime}$ which is a natural transformation $\left\{\eta_{j}\right\}_{j \in J}=\left\{d(j) \Longrightarrow d^{\prime}(j)\right\}_{j \in J}$ from the functor $d$ to a functor $d^{\prime}$ and where the commutativity properties of the natural transformation insure that the composition $c \rightarrow d \Longrightarrow d^{\prime}$ of a het $c \rightarrow d$ followed by the $C^{J}$-hom $d \Longrightarrow d^{\prime}$ is another het-cone $c \rightarrow d^{\prime}$.

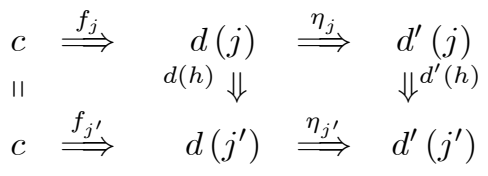

With the Het-bifunctor "between" the categories and the Hom-bifunctors within each category handling all the compositions, the universal mapping property characterizing the lim-functor is stated as a right representation without any $\Delta$-device:

$$
\operatorname{Het}(c, d) \cong \operatorname{Hom}_{C}(c, \lim (d)) .
$$

Taking $c=\lim (d)$, the correspondence picks out the universal cone [16, p. 72] $\lim (d) \stackrel{\varepsilon_{d}}{\rightarrow} d$ so the right representation is then given by the device-free diagram.

$$
c \underset{\searrow^{\varphi}}{\stackrel{\exists ! \bar{f}}{\Longrightarrow}} \lim (d)
$$

Right representation characterizing

the lim-functor without the diagonal functor $\Delta$.

But the $\Delta$ device has a left representation:

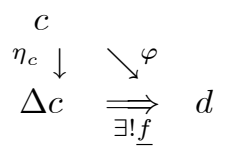

which is expressed as the isomorphism natural in $d:{ }^{11}$

$$
\operatorname{Hom}_{C^{J}}(\Delta c, d) \cong \operatorname{Het}(c, d)
$$

where $\eta_{c}$ is the universal cone $c \rightarrow \Delta c$ obtained in the left representation isomorphism from $1_{\Delta c}$ after taking $d=\Delta c$.

\begin{tabular}{|c|c|c|}
\hline$d \stackrel{\varepsilon_{d}}{\Longleftarrow}$ & $\Delta(\lim (d)$ & $\lim (d)$ \\
\hline 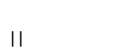 & $\Delta(\bar{f})_{\Uparrow}$ & $\exists ! \bar{f} \Uparrow$ \\
\hline$d \stackrel{f}{\Longleftarrow}$ & $\Delta(c)$ & $c$ \\
\hline
\end{tabular}

Using Mac Lane's device of a co-universal arrow from the diagonal functor $\Delta$ to $d$, the limit functor $\lim ()$ would satisfy the homs-only over-and-back adjunction diagram (where we used the customary Greek $\varepsilon$ for the $C^{J}$-hom $\left.\Delta(\lim (d)) \Longrightarrow d\right)$.

Diagram for het-free co-universal arrow

\footnotetext{
${ }^{11}$ It should be noted that many authors routinely switch back and forth between the het cone $\varphi: c \rightarrow d$ going from $c \in C$ to $d \in C^{J}$, and the left representation hom $f: \Delta(c) \Rightarrow d$ inside $C^{J}$.
} 
With the diagonal functor giving a left representation, we have the heteromorphic diagram for the adjunction given by putting the left and right representation diagrams together at their common diagonal het $c \rightarrow d$ :

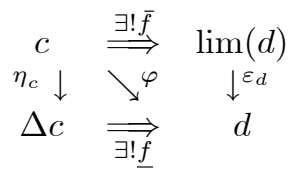

Adjunctive square diagram for the diagonal and limit adjoints

and we have the natural equivalences obtained by putting the left and right representation isomorphisms together at their common middle term $\operatorname{Het}(c, d)$ :

$$
\operatorname{Hom}_{C^{J}}(\Delta c, d) \cong \operatorname{Het}(c, d) \cong \operatorname{Hom}_{C}(c, \lim (d)) \text {. }
$$

In spite of the diagonal functor not being necessary to state the universal mapping property for limits, it does have a role in addressing another question. Given the hets going one way between two categories, an adjunction is the bi-representation of those hets in the domain and codomain categories. But what about the hets going the other way? There is sometimes a similar bi-representation of those hets where the diagonal is again one of the functors [20, pp. 87-8]. Indeed, in the case at hand, the hets $\varphi: C \ni c \rightarrow d \in C^{J}$ are the cones and the hets going the other way are the cocones $\gamma: C^{J} \ni d \rightarrow c \in C$ and the non-trivial representing functor in the codomain category gives the colimits.

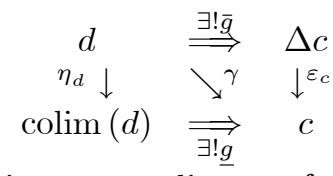

Adjunctive square diagram for colimits

The adjunctive square diagrams for the limit and the colimit can then be combined at the common corner for $\Delta c$.

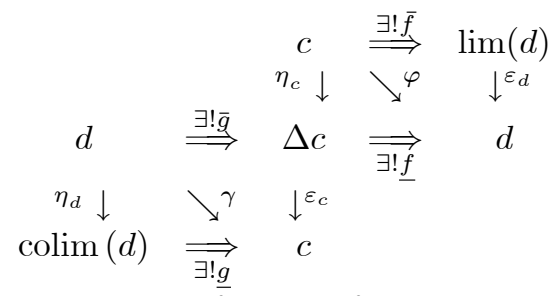

Adjunctive squares for the left and right adjoints

to the diagonal functor overlapping at $\Delta c$.

\section{Adjoints vs. UMPs: What's basic?}

There is a growing consensus among category theorists that category theory has foundational importance not simply because it provides the language in which to formulate mathematics (like set theory) but because it provides the conceptual lens (as set theory does not) to put into focus the important parts of mathematics. And adjoints are widely perceived as the basic lens (Lawvere [15]). As Steven Awodey put it:

The notion of adjoint functor applies everything that we have learned up to now to unify and subsume all the different universal mapping properties that we have encountered, from free groups to limits to exponentials. But more importantly, it also captures an important mathematical phenomenon that is invisible without the lens of category theory. Indeed, I will make the admittedly provocative claim that adjointness is a concept of fundamental logical and mathematical importance that is not captured elsewhere in mathematics. [1, p. 179] 
Other category theorists have given similar testimonials.

To some, including this writer, adjunction is the most important concept in category theory. (Wood $[29$, p. 6])

The isolation and explication of the notion of adjointness is perhaps the most profound contribution that category theory has made to the history of general mathematical ideas. (Goldblatt $[10$, p. 438])

Nowadays, every user of category theory agrees that [adjunction] is the concept which justifies the fundamental position of the subject in mathematics. (Taylor [28, p. 367])

The Mac Lane-Bourbaki-Samuel episode raises a more basic question: should adjoints be taken as that basic conceptual lens, or should an adjunction be seen as a molecular concept that can be split into the two atoms of solutions to the universal and co-universal mapping problems? Heteromorphisms seem to be key to that question since, as we have argued, the "natural" or clean formulation of a universal mapping problem is as a heteromorphic representation problem-in contrast, say, to Mac Lane's convoluted over-andback homs-only formulations of the universal arrows or universal elements-although ultimately each concept "subsumes" (Mac Lane [20, p. 61]) the others.

Given a bifunctor Het : $X^{o p} \times A \rightarrow$ Set "connection" ([25, p. 58]) or "correspondence" (Lurie [17, p. 96]) from $X$ to $A$, adjoints arise as the left and right representations of the bifunctor, and conversely, by the "Adjunction Representation Theorem" [9, p. 147]:

given any adjunction $F: X \rightleftarrows A: G$, heteromorphisms can be defined between (isomorphic copies of) the categories $X$ and $A$ so that (isomorphic copies of) the adjoints arise from the representations on the left and right of the het-bifunctor. (Ellerman $[9$, p. 130])

Thus all adjunctions arise as bi-representations of Het-bifunctors but there can be either a left or right representation without the other (e.g., the tensor product example). Moreover, when one of a pair of adjoints gives the important concept and the other adjoint functions as an auxiliary device to fill out the het-free notion of an adjunction, then the important left or right representation is routinely used in the literature (along with the necessary hets) without the auxiliary device. These considerations argue that it is the concept of a universal mapping property (which is naturally formulated as a representation of hets) that is the "most important concept in category theory" and that adjunctions arise as the special case of $b i$-representations of hets.

Since this treatment of UMPs is based on the notion of a representation of a functor associated with Alexander Grothendieck ([11, representable functors are defined in first section of the first Chapter 0]), it helps to clear up another mystery.

As we can see by looking at his [Grothendieck's] lectures in the Séminaire Bourbaki from 1957 until 1962, the notion of representable functors became one of the main tools he used... . It is far from clear why Grothendieck decided to use this notion instead of, say, adjoint functors,... . It is also clear from the various seminars that Grothendieck thought in terms of universal "problems", that is he tried to formulate the problems he was working on in terms of a universal morphism: finding a solution to the given problem amounted to finding a universal morphism in the situation. Grothendieck saw that the latter notion was subsumed under the notion of representable functor. [22, p. 102-3]

In short, Grothendieck took as basic the notion of universal problem or UMP formulated as a representation, and then adjoints arise as the special case of a particularly nice bi-representation. To personalize the "adjoints vs. UMPs" issue, perhaps it is: Mac Lane vs. Grothendieck. 


\section{Conclusions}

We have revisited, from a heteromorphic viewpoint, Mac Lane's famous remarks about how Bourbaki (Samuel) "just missed" adjoints. But after Mac Lane category-theoretically reformulated what Samuel had done using a Het-bifunctor $W: X^{o p} \times A \rightarrow$ Set, we see that Samuel defined a left representation $\operatorname{Hom}_{A}(F(x), a) \cong$ $W(x, a)$. Dualizing yields the notion of a right representation $W(x, a) \cong \operatorname{Hom}_{X}(x, G(a))$ so putting the two isomorphisms together, we have the heteromorphic adjunction equivalence:

$$
\operatorname{Hom}_{A}(F(x), a) \cong W(x, a) \cong \operatorname{Hom}_{X}(x, G(a))
$$

which seems to be first developed by Pareigis in the late 1960's. Hence, Samuel did miss adjoints but (reformulated in categorical terms) he was only a simple dualization away from defining an adjunction a decade before Kan.

These observations prompted some analysis of the puzzling reticence in orthodox category theory to formally recognize the object-to-object morphisms for objects in different categories. Some artificiality and various devices are employed to avoid any official use of hets although hets are commonly used informally by working category theorists, not to mention other working mathematicians. Since hets can be rigorously treated using Het-bifunctors in a way quite analogous to the usual Hom-bifunctors, the usual heterophobia seems not to be based on any mathematical concerns for rigor. In view of the analogy with the Erlangen Program, the founders only considered homomorphisms, so one rationale may be respect for the founders' original vision. Or it may just be the force of habit or intellectual inertia so that change again awaits those "less beholden to tradition or to fashion."

Finally we considered the "philosophical" question that lurks in the background about the "most important concept in category theory": is it the notion of an adjunction or the notion of a universal mapping property (cleanly formulated as a representation of hets)?

\section{References}

[1] Awodey, Steve. 2006. Category Theory. Oxford: Clarendon Press.

[2] Bénabou, J. 1973. Les distributeurs. Report 33. Université Catholique de Louvain: Institut de Mathématique Pure et appliquée.

[3] Bourbaki, Nicolas. 1948. Elements de Mathematique, Vol. VII., Algebre, Livre II, Algebre Multilineaire, Ch. 3. Vol. VII. Actualites scientifiques et industrielles, 1044. Paris: Hermann.

[4] Bourbaki, Nicolas. 1968. Theory of Sets. Reading MA: Addison-Wesley.

[5] Bourbaki, Nicolas. 1974. Elements of Mathematics: Algebra I Chapters 1-3. Reading MA: AddisonWesley.

[6] Corry, Leo. 2004. Modern Algebra and the Rise of Mathematical Structures, Second Revised Ed. Basel: Springer Basel AG.

[7] Eilenberg, Samuel, and Saunders Mac Lane. 1945. General Theory of Natural Equivalences. Transactions of the American Mathematical Society. 58 (2): 231-94.

[8] Ellerman, David 1988. Category Theory and Concrete Universals. Erkenntnis. 28: 409-29.

[9] Ellerman, David. 2006. A Theory of Adjoint Functors-with Some Thoughts on Their Philosophical Significance. In What Is Category Theory?, edited by G. Sica, 127-83. Milan: Polimetrica.

[10] Goldblatt, Robert 2006 (1984). Topoi: the Categorical Analysis of Logic (revised ed.). Mineola NY: Dover. 
[11] Grothendieck, Alexander, and Jean Dieudonné. 1971. Eléments de Géométrie Algébrique. Berlin: Springer-Verlag.

[12] Jacobson, Nathan. 1985. Basic Algebra I. 2nd ed. New York: W.H. Freeman.

[13] Kan, Daniel 1958. Adjoint Functors. Transactions of the American Mathematical Society. 87 (2): 294329.

[14] Kelly, Max. 1982. Basic Concepts of Enriched Category Theory. Cambridge: Cambridge University Press.

[15] Lawvere, F. William 1969. Adjointness in Foundations. Dialectica. 23: 281-95.

[16] Lawvere, F. William, and Robert Rosebrugh. 2003. Sets for Mathematics. Cambridge: Cambridge University Press.

[17] Lurie, Jacob. 2009. Higher Topos Theory. Princeton NJ: Princeton University Press.

[18] Mac Lane, Saunders. 1948. Groups, Categories, and Duality. Proc. Nat. Acad. Sci. U.S.A. 34 (6): 263-67.

[19] Mac Lane, Saunders. 1949. Review of: Bourbaki, N. Elements de Mathematique. VII. Premiere Partie: Les Structures Fondamentales de L'analyse. Livre II: Algebre. Chapitre III: Algebre Multilineaire. (French) Actualites Sci. Ind., No. 1044. Hermann et Cie., Paris, 1948. Mathematical Reviews, no. MR0026989 (10,231d). http://www.ams.org/mathscinet/pdf/26989.pdf.

[20] Mac Lane, Saunders. 1998. Categories for the Working Mathematician. 2nd Ed. New York: Springer Science+Business Media.

[21] Mac Lane, Saunders, and Garrett Birkhoff. 1988. Algebra. Third edition. New York: Chelsea.

[22] Marquis, Jean-Pierre. 2009. From a Geometrical Point of View: A Study of the History and Philosophy of Category Theory. Berlin: Springer Science+Business Media.

[23] McLarty, Colin. 1992. Elementary Categories, Elementary Toposes. Oxford: Clarendon Press.

[24] McLarty, Colin. 2005. Saunders Mac Lane (1909-2005): His Mathematical Life and Philosophical Works. Philosophia Mathematica (III), 13: 237-51.

[25] Pareigis, Bodo. 1970. Categories and Functors. New York: Academic Press.

[26] Samuel, Pierre. 1948. On Universal Mappings and Free Topological Groups. Bull. Am. Math. Soc. 54 (6): 591-98.

[27] Shulman, Mike. 2011. Cograph of a Profunctor. Ncatlab.org. October 31. http://ncatlab.org/nlab/show/cograph+of+a+profunctor.

[28] Taylor, Paul 1999. Practical Foundations of Mathematics. Cambridge UK: Cambridge University Press.

[29] Wood, Richard J. 2004. Ordered Sets via Adjunctions. In Categorical Foundations. Encyclopedia of Mathematics and Its Applications Vol. 9\%. Maria Cristina Pedicchio and Walter Tholen eds., Cambridge: Cambridge University Press: 5-47. 